\title{
Sub-nanometric Manganous Oxide Clusters in Nitrogen Doped Mesoporous Carbon Nanosheets for High-Performance Lithium-Sulfur Batteries
}

Pengpeng Qiu ${ }^{\mathrm{a}}$, Yu Yao ${ }^{\mathrm{b}}$, Wei Li ${ }^{\mathrm{c} *}$, Yang Sun ${ }^{\mathrm{d}}$, Zheng Jiange,f, Bingbao Meie,f, Lin

$\mathrm{Gu}^{\mathrm{g}}$, Qinghua Zhangs, Tongtong Shang ${ }^{\mathrm{g}}$, Xiqian $\mathrm{Yu}^{\mathrm{h}}$, Jianping Yang ${ }^{\mathrm{a}}$, Yuan Fang ${ }^{\mathrm{a}}$, Guihua Zhu', Ziling Zhang ${ }^{\mathrm{a}}$, Xiaohang Zhu ${ }^{\mathrm{a}}$, Tao Zhao ${ }^{\mathrm{a}}$, Wan Jianga, Yuchi Fan ${ }^{\mathrm{a}}$, Lianjun Wang ${ }^{\mathrm{a}}$, Bin $\mathrm{Ma}^{\mathrm{c}}$, Liangliang $\mathrm{Liu}^{\mathrm{c}}$, Yan $\mathrm{Yu}^{\mathrm{b} *}$, and Wei Luo ${ }^{\mathrm{a} *}$

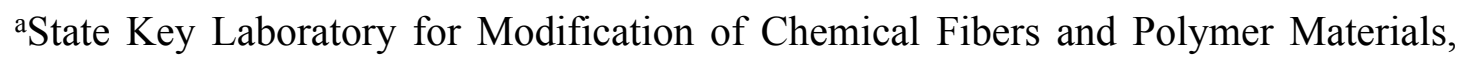

College of Materials Science and Engineering, Institute of Functional Materials, Donghua University, Shanghai 201620, China.wluo@dhu.edu.cn

${ }^{b}$ Hefei National Laboratory for Physical Sciences at the Microscale, Department of Materials Science and Engineering, CAS Key Laboratory of Materials for Energy Conversion, University of Science and Technology of China, Hefei, Anhui 230026, China. yanyumse@ustc.edu.cn

'Department of Chemistry, Shanghai Key Laboratory of Molecular Catalysis and Innovative Materials, Fudan University, Shanghai 200433, China. weilichem@fudan.edu.cn

dSchool of Materials, Sun Yat-sen University, Guangzhou 510006, China

eShanghai Synchrotron Radiation Facility, Zhangjiang Lab, Shanghai Advanced Research Institute, Chinese Academy of Science, Shanghai 201213, China

fShanghai Institute of Applied Physics, Chinese Academy of Sciences, Shanghai 201204, China 
gBeijing National Laboratory for Condensed Matter Physics, Institute of Physics, Chinese Academy of Sciences, Beijing, 100190, China

hBeijing Advanced Innovation Center for Materials Genome Engineering, Institute of Physics, Chinese Academy of Sciences, Beijing, 100190, China 


\section{Experimental section.}

\section{Chemicals and Materials.}

PEO-5000 were purchased from Sigma-Aldrich. 2-bromoisobutyryl bromide, N, N, N', N', N'-Pentamethyldiethylenetriamine (PMDETA) was purchased from Acros. Styrene, tetrahydrofuran (THF) ( $>99 \%)$, pyridine $(>99 \%)$, cuprous bromide $(\mathrm{CuBr})$, ammonium hydroxide $\left(\mathrm{NH}_{4} \mathrm{OH}, 28-30\right.$ wt \%), dopamine hydrochloride, manganese acetate and ethanol were purchased from Shanghai Chemical Corp. All chemicals were of analytical grade and used without further purification. Deionized water was used for all experiments.

\section{Synthesis of PEO-b-PS diblock copolymer}

The PEO-b-PS diblock copolymer was prepared by an atom transfer radical polymerization (ATRP) method, which involves two steps. In the first step, the microinitiator PEO-Br was prepared through the acyl bromide reaction. $20.0 \mathrm{~g}$ of PEO-5000 was dissolved in $120 \mathrm{~mL}$ of THF. Then, $20 \mathrm{~mL}$ of pyridine was added to form a homogeneous solution at $30^{\circ} \mathrm{C}$, which was followed reducing the temperature to $0{ }^{\circ} \mathrm{C}$ by using an ice-water bath. Next, $6.00 \mathrm{~g}$ of 2-bromoisobutyryl bromide was added dropwise, and the mixed solution was stirred at $30^{\circ} \mathrm{C}$ for $24 \mathrm{~h}$. After adding $200 \mathrm{~mL}$ of cold ether, the white precipitation of PEO-Br was collected by centrifugation, washed with cold ether, and dried in vacuum at $30{ }^{\circ} \mathrm{C}$ overnight. In the second step, $5.00 \mathrm{~g}$ of the above made PEO-Br, $0.15 \mathrm{~g}$ of $\mathrm{CuBr}, 0.50 \mathrm{~g}$ of PMDETA and $35 \mathrm{~mL}$ of styrene were added to an ampoule bottle and degassed with three freeze-pump-thaw cycles. The mixture was subsequently immersed in an oil bath at $115^{\circ} \mathrm{C}$ under stirring. 
After reacting for $2 \mathrm{~h}$, the ampoule bottle was cooled down in liquid nitrogen. The gellike product was dissolved in $100 \mathrm{~mL}$ of THF and filtered through $\mathrm{Al}_{2} \mathrm{O}_{3}$ column to remove $\mathrm{Cu}$ complex. After that, $200 \mathrm{~mL}$ of petroleum ether was poured into the solution to precipitate PEO-b-PS block copolymer. The copolymer was then dried in vacuum at $40{ }^{\circ} \mathrm{C}$.

\section{Synthesis of rGO@mC-MnO-800 nanosheets.}

In a typical synthesis, $60 \mathrm{mg}$ of PEO- $b$-PS was dissolved in $8 \mathrm{ml}$ of THF to obtain a transparent solution. Then, a mixture of water/ethanol solution $(24 / 12 \mathrm{ml})$ was quickly added to the solution to obtain a light-blue micelle colloid solution, which presents clear Tyndall effect. After stirring for $5 \mathrm{~min}, 80 \mathrm{mg}$ of dopamine (DP) and $10 \mathrm{mg}$ of manganese acetate were added. Owing to the slight oxidative ability of $\mathrm{Mn}^{2+}$ ions, the solution gradually become gray with string for $5 \mathrm{~min}$. Then, $0.5 \mathrm{ml}$ of GO solution ( 5 $\mathrm{mg} / \mathrm{ml}$ ) prepared by a modified Hummers method was injected into the solution above. After sonicating the solution for $10 \mathrm{~min}$, the reaction was initiated by adjusting the $\mathrm{pH}$ to 8 using a certain amount of ammonia hydroxide. After reacting for $8 \mathrm{~h}$, the as-made sample were obtained by centrifuging at $8000 \mathrm{rpm}$ for $5 \mathrm{~min}$ and washed repeatedly for three time by using water and ethanol, respectively. Finally, the obtained black precipitates were freeze dried and then carbonized at $800{ }^{\circ} \mathrm{C}$ in $\mathrm{N}_{2}$ atmosphere.

As a comparison, the $\mathrm{rGO} @ \mathrm{mC}-800$ was prepared without the addition of manganese acetate. The rGO@mC-MnO-900 was synthesized by annealing the asmade sample in $900{ }^{\circ} \mathrm{C}$

\section{Synthesis of the S-based Cathodes}


The S-based cathodes were prepared via the melt-diffusion method. Typically, 30 mg sulfur powder was mixed with 20 mg rGO@mC-MnO-800 with heat treatment at $155^{\circ} \mathrm{C}$ for $12 \mathrm{~h}$ in a sealed glass vessel under $\mathrm{Ar}$ atmosphere to gain $\mathrm{S} / \mathrm{rGO} @ \mathrm{mC}-\mathrm{MnO}-$ 800 cathode. Similarly, the S/rGO@mC-MnO-900 and S/rGO@mC cathodes were obtained by the same way.

\section{Characterizations.}

TEM was carried out (JEOL 2011, Japan) at $200 \mathrm{kV}$. FESEM images were obtained using field emission scanningmicroscopy (Hitachi model S-4800, Japan). XRD patterns were collected (Bruker D4, Germany) with Ni-filtered Cu KR radiation (40kV, $40 \mathrm{~mA})$. The $\mathrm{N}_{2}$ sorption isotherms were measured at $77 \mathrm{~K}$ (Micromeritcs Tristar 3020, USA). Before measurement, the samples were degassed in a vacuum at $180^{\circ} \mathrm{C}$ for at least $8 \mathrm{~h}$. The surface areas were calculated by the BET method using the adsorption data in a relative pressure $(\mathrm{P} / \mathrm{P} 0)$ ranged from 0.005 to 0.25 . By using the $\mathrm{BJH}$ method, the pore size distribution and pore volume were calculated from the adsorption branches of isotherms, and the total pore volume $(\mathrm{Vt})$ was estimated from the adsorbed amount at the relative pressure $\left(\mathrm{P} / \mathrm{P}_{0}\right)$ of 0.995 . XPS was collected with a monochrome $\mathrm{Al}$ source (AXIS ULTRA DLD XPS Shimadzu Corp.). All of the binding energies were modified using the $\mathrm{C} 1 \mathrm{~s}$ standard peak at $284.6 \mathrm{eV}$. XAFS measurements at Mn K-edge in fluorescence mode with a Lytle detector were performed at the BL14W1 in Shanghai Synchrotron Radiation Facility (SSRF) [1]. The electron beam energy was $3.5 \mathrm{GeV}$ and the stored current was $220 \mathrm{~mA}$ (top-up). A 38-pole wiggler with the maximum magnetic 
field of $1.2 \mathrm{~T}$ inserted in the straight section of the storage ring was used. XAFS data were collected using a fixed-exit double-crystal Si (111) monochromator. The energy was calibrated using Mn foil. The photon flux at the sample position was $2.6 \times 1012$ photons per second. The raw data analysis was performed using IFEFFIT software package according to the standard data analysis procedures [2]. The spectra were calibrated, averaged, pre-edge background subtracted, and post-edge normalized using Athena program in IFEFFIT software package. The Fourier transformation of the $\mathrm{k}^{3}-$ weighted EXAFS oscillations, $\mathrm{k}^{3} \bullet \chi(\mathrm{k})$, from $\mathrm{k}$ space to $\mathrm{R}$ space was performed to obtain a radial distribution function. And data fitting was done by Artemis program in IFEFFIT.

\section{Electrochemical Investigation}

2032-type coin cells were used to test the electrochemical performance of cathode materials with lithium metal as reference electrodes and Celgard 2400 membrane as the separator. Cathode materials were composed of $80 \%$ active materials, $10 \%$ polyvinylidenedifluoride (PVDF) and 10\% carbon black. The electrolyte was composed of $1 \mathrm{M}$ lithium bis(trifluoromethanesulfonyl)imide (LiTFSI) in a solvent mixture of 1,3-dioxolane (DOL) and dimethoxymethane (DME) (1:1 in volume) with $2 \mathrm{wt} \% \mathrm{LiNO}_{3}$ as an additive. The areal $\mathrm{S}$ loading was about $1.5 \mathrm{mg} \mathrm{cm} \mathrm{cm}^{-2}$. All the capacity values were calculated based on the mass of S. The galvanostatic electrochemical measurements were carried out in the Neware BTS-610 instrument with the voltage window of 1.6-2.8 V. The cyclic voltammetry $(\mathrm{CV})$ test was performed 
in a scan rate of $0.2 \mathrm{mV} \mathrm{s}^{-1}$ and the electrochemical impedance spectrum (EIS) measurements were obtained in the frequency range of $100 \mathrm{kHz}$ to $0.01 \mathrm{~Hz}$ on the $\mathrm{CHI}$ 660D workstation.

\section{Computational Details}

Density functional theory (DFT) calculations were performed using the Vienna $\mathrm{Ab}$ initio Simulation Package (VASP) within the projector augmented-wave (PAW) formalism [3-5]. The Perdew-Burke-Ernzerhof (PBE) parameterised generalized gradient approximation (GGA) pseudo-potential was used to describe the exchangecorrelation potential [6]. The van der Waals (vdW) interaction was taken into account by using the rev-vdW-DF2 functional [7-9]. The $\operatorname{Li}(1 s, 2 s, 2 p), \mathrm{S}(3 s, 3 p), \mathrm{C}(2 s, 2 p)$, $\mathrm{Mn}(3 d, 4 s)$, and $\mathrm{O}(2 s, 2 p)$ orbitals are treated as valence states. The plane-wave cutoff was set to $400 \mathrm{eV}$. Geometry optimizations were performed by using a conjugate gradient minimization until all the forces acting on ions were less than $0.01 \mathrm{eV} / \AA$ per atom. The $\Gamma$ point was used in the Brillouin zone sampling to ensure a feasible computational cost. The substrate is a $6 \times 6$ graphene sheet, separated by a vacuum layer of $20 \AA$. The adsorption energy between the surface and adsorbed molecule is determined by $E_{\mathrm{b}}=E_{\text {surf. }}+E_{\text {ads. }}-E_{\text {ads.@surf., where }} E_{\text {surf., }} E_{\text {ads., }}$ and $E_{\text {ads.@surf. denote the }}$ total energies of the pristine surface, the adsorbed molecule, and the adsorbed surface, respectively. 


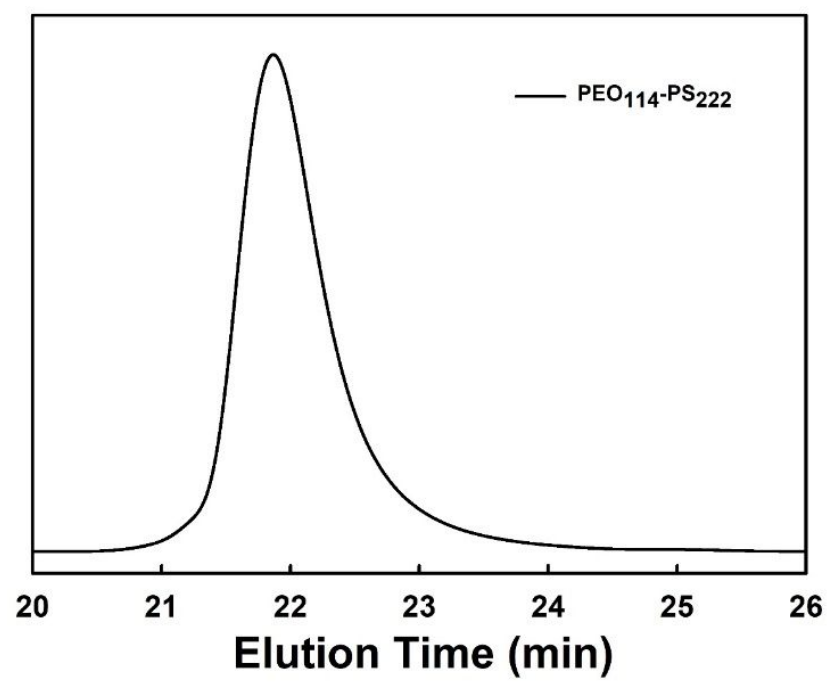

Figure S1. Gel permeation chromatography traces of $\mathrm{PEO}_{114}-\mathrm{b}-\mathrm{PS}_{222}$. 

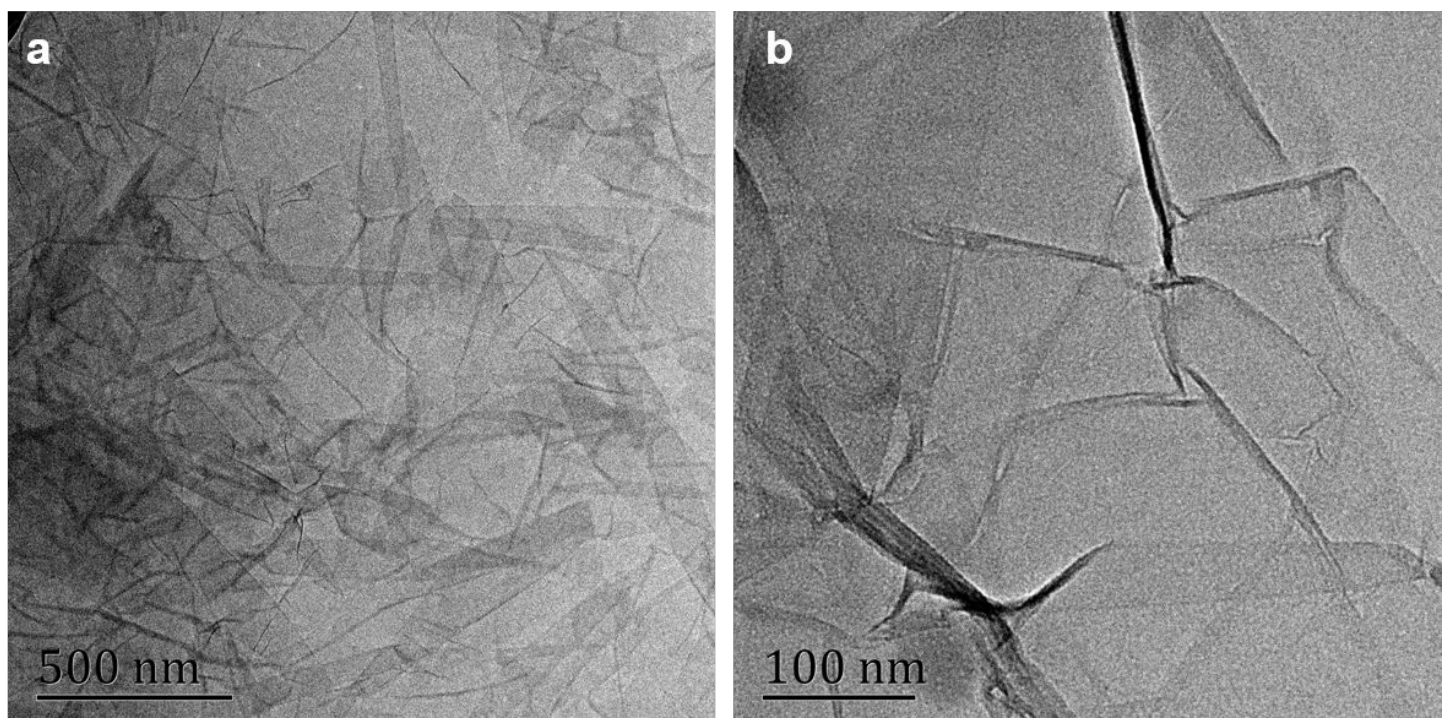

Figure S2. TEM images of GO synthesized through the modified hummer method. 

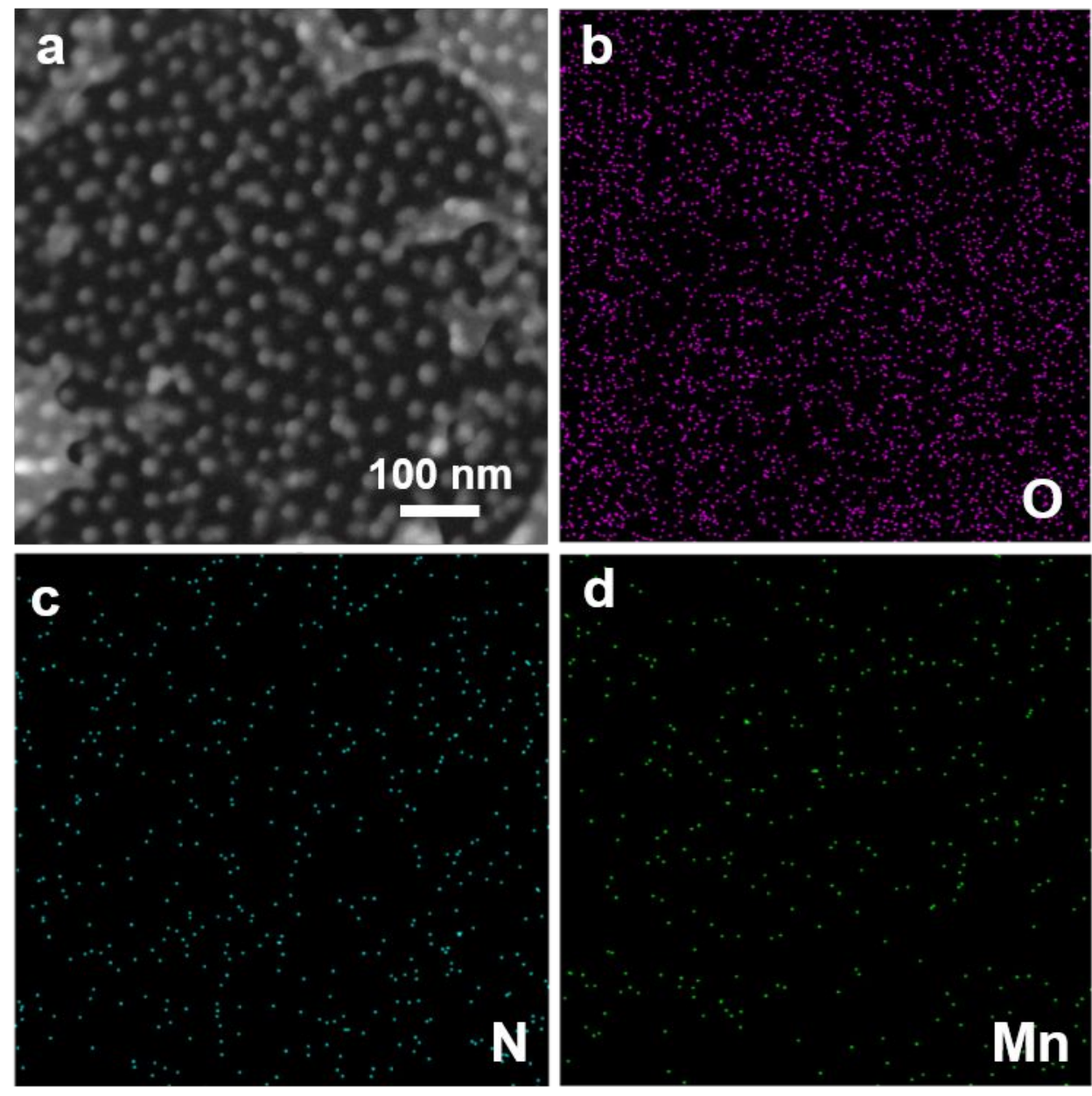

Figure S3. STEM image of the PEO- $b$-PS/DA-Mn composite micelles (a) and its corresponding elemental mapping of oxygen (b), nitrogen (c), and manganese (d). 


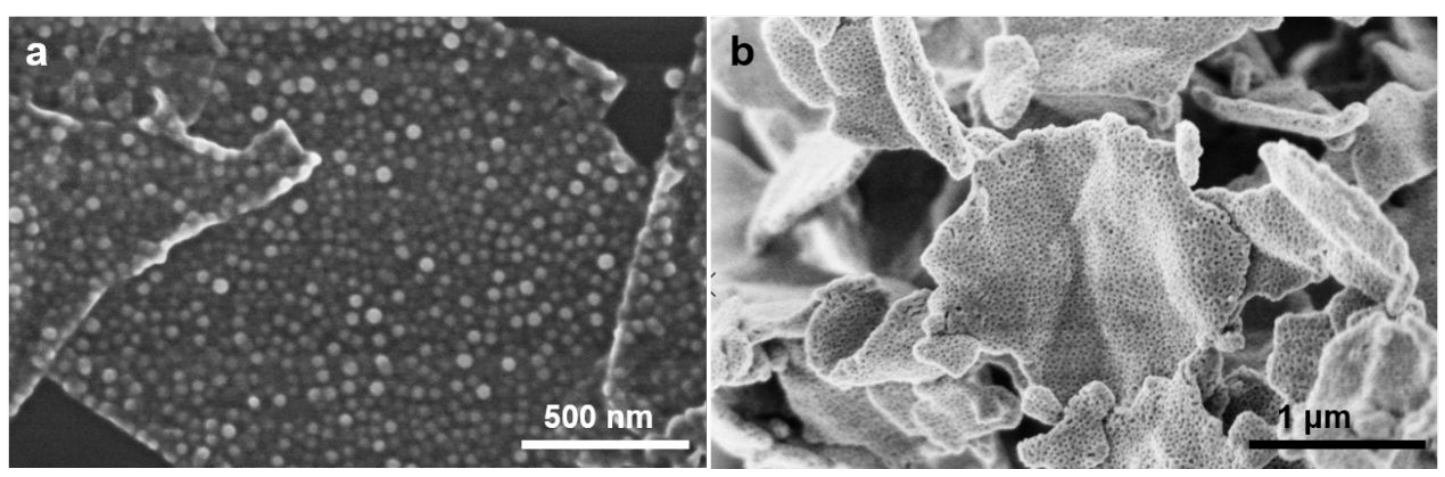

Figure S4. SEM images of the as-made GO@PEO- $b$-PS/Mn-DA (a) and rGO@mC$\mathrm{MnO}-800$ (b). 


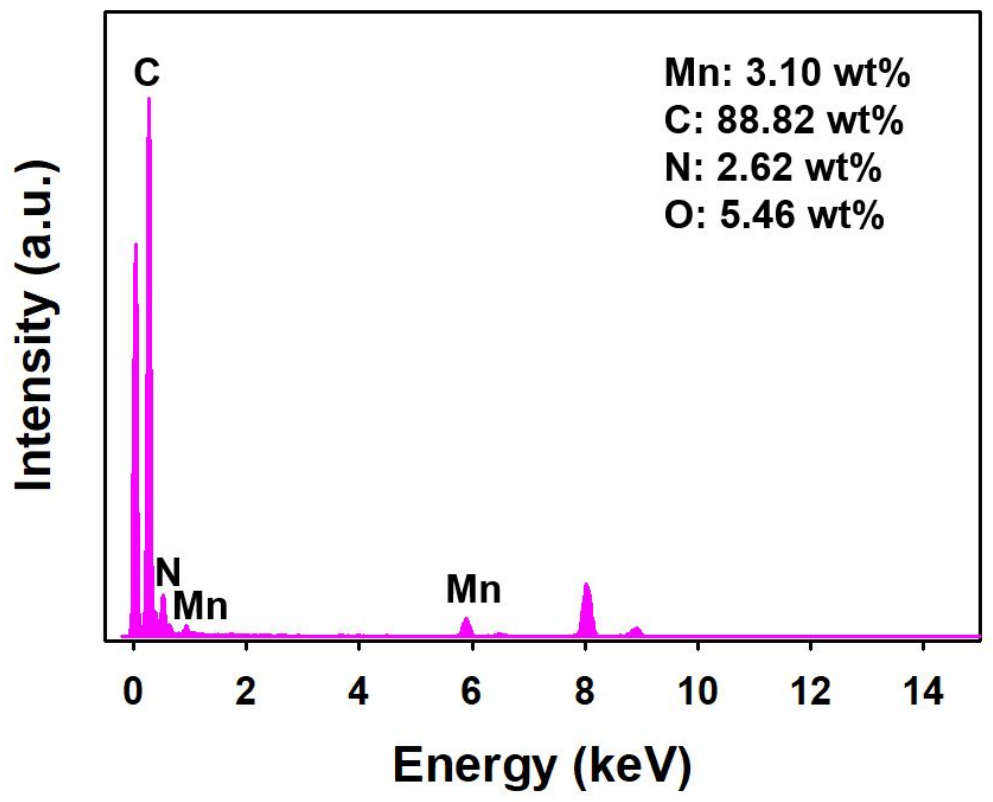

Figure S5. The EDS analysis of the rGO@mC-MnO-800 sample. 

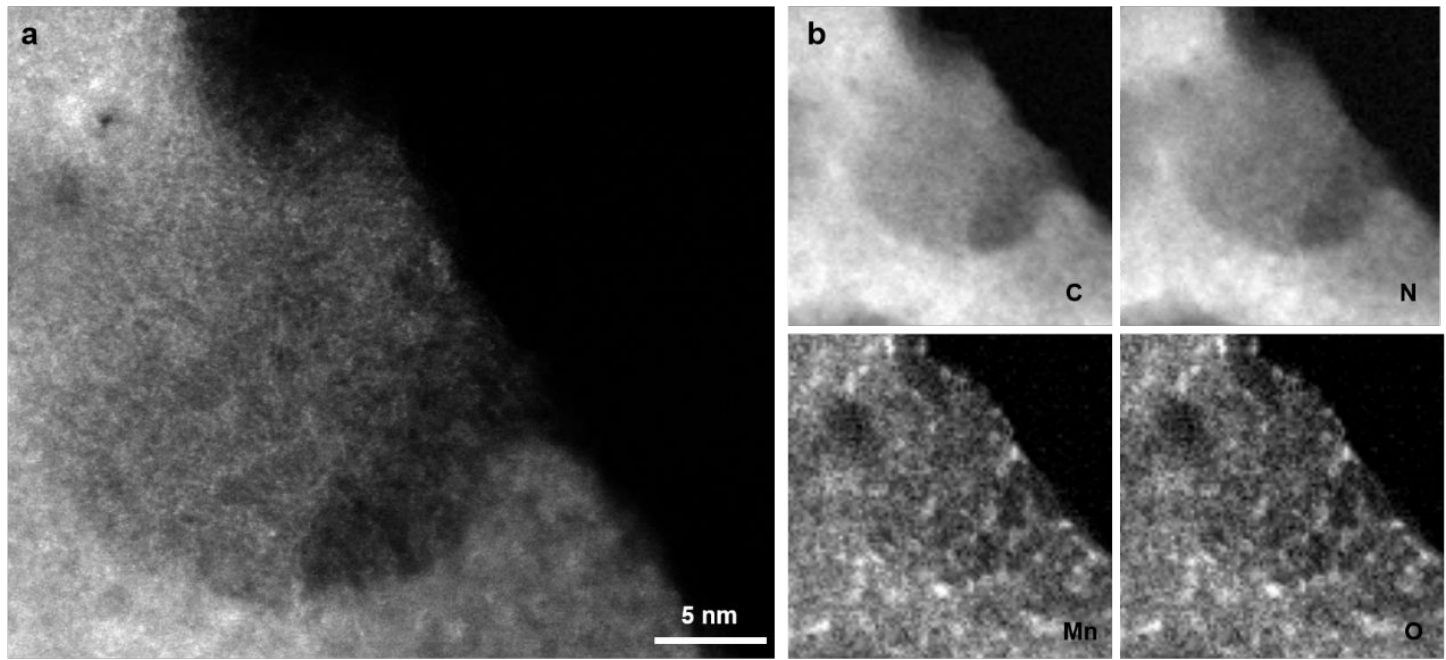

Figure S6. Low-magnification HAADF-STEM image of rGO@mC-MnO-800 and its corresponding electron energy loss spectroscopy of C, N, Mn and O (b). 

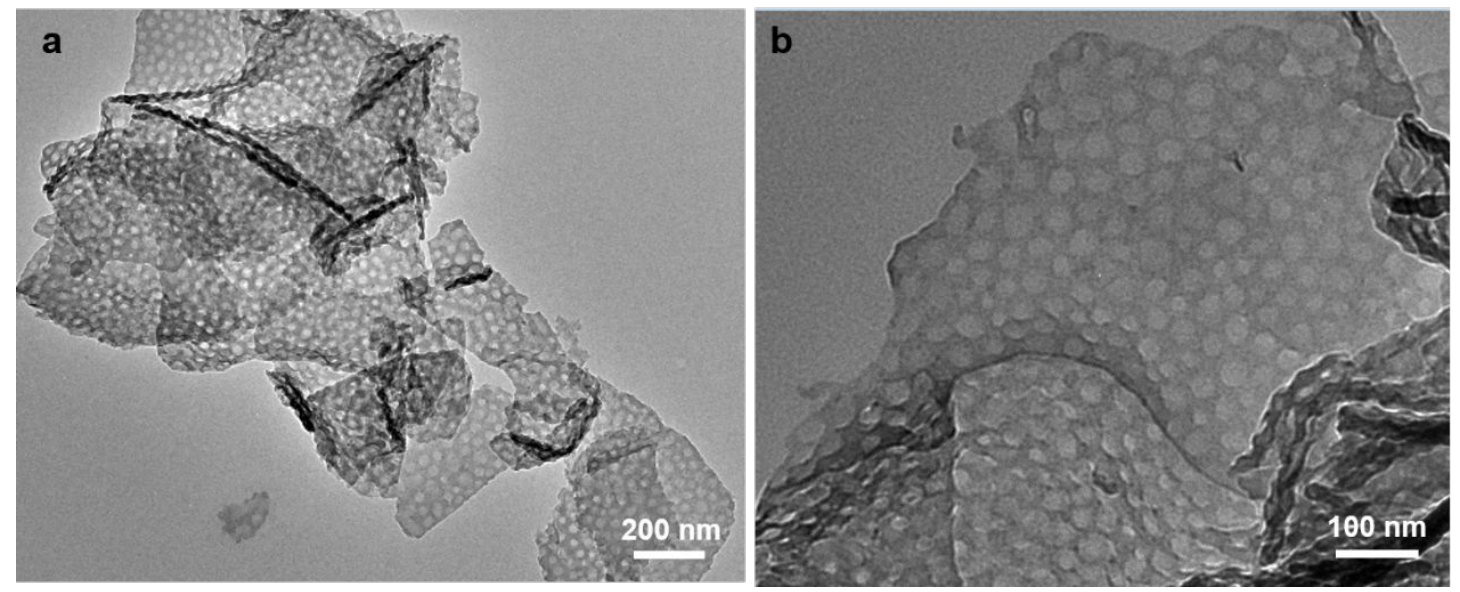

Figure S7. TEM images of the rGO@mC-800. 

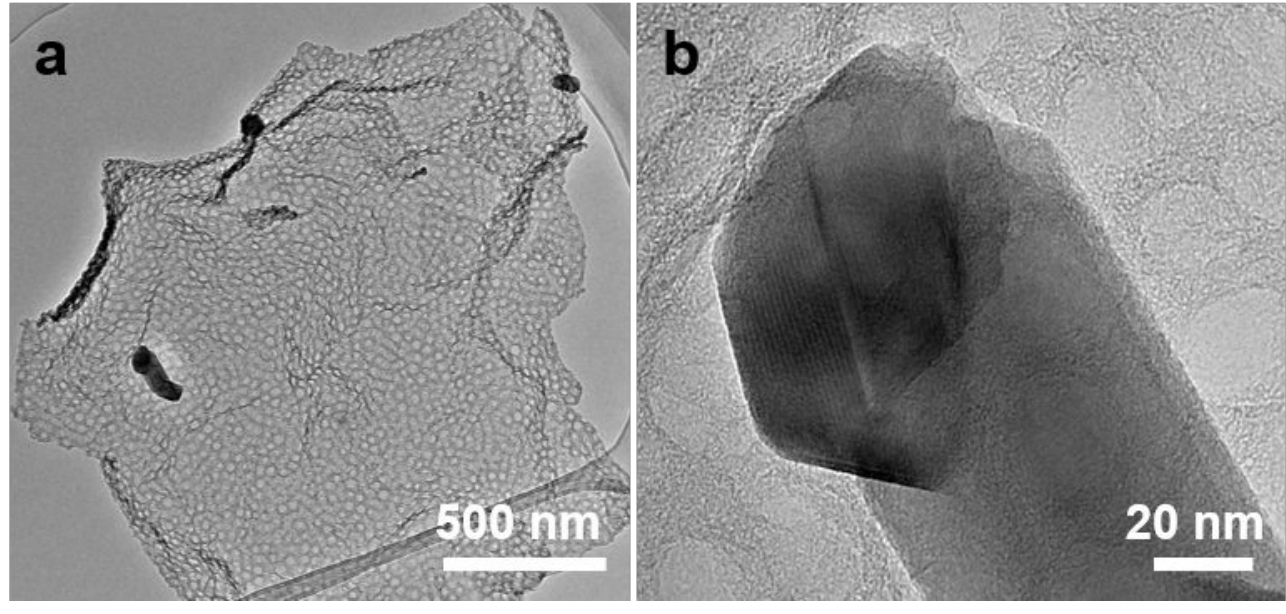

Figure S8. TEM (a) and HRTEM (b) images of the rGO@mC-MnO-900. 


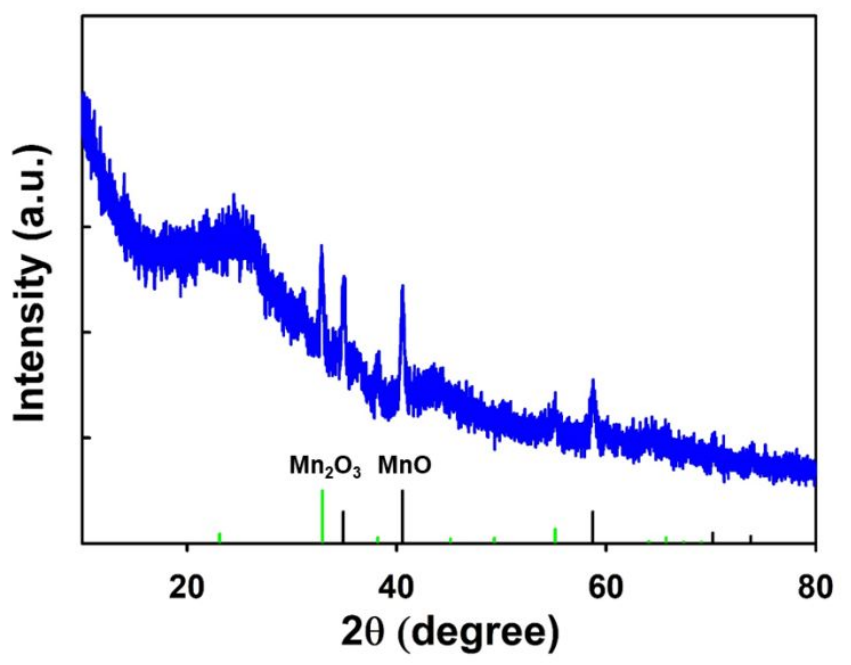

Figure S9. The XRD pattern of the rGO@mC-MnO-900. 


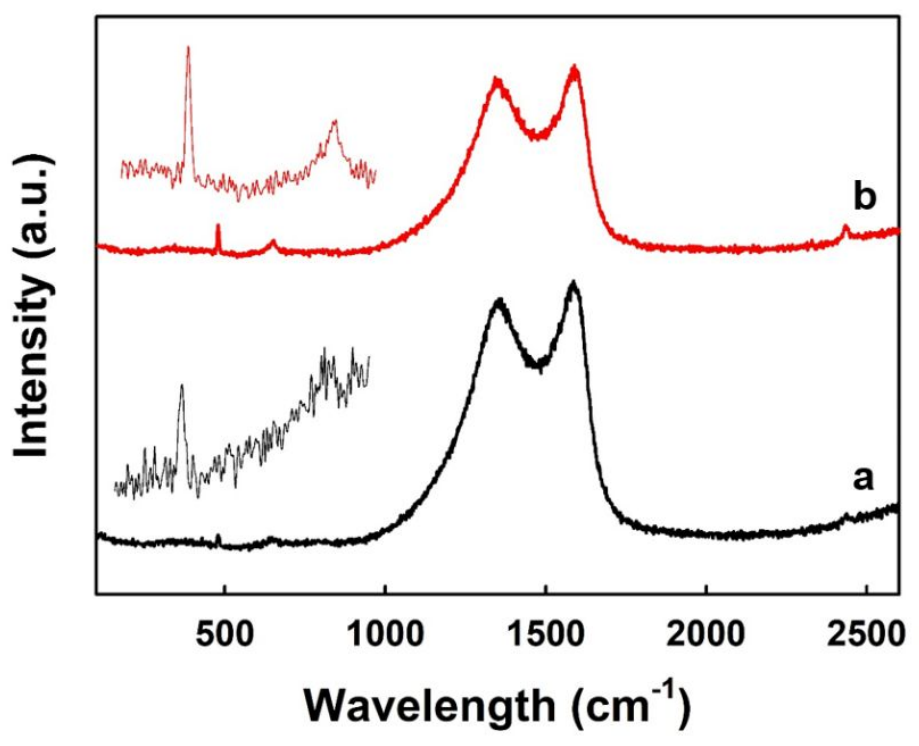

Figure S10. Raman spectra of the rGO@mC-MnO-800 (a) and rGO@mC-MnO-900 (b). Figure insert are enlarged spectra (10 times) from the wavelength of 490 to 505 $\mathrm{cm}^{-1}$ 

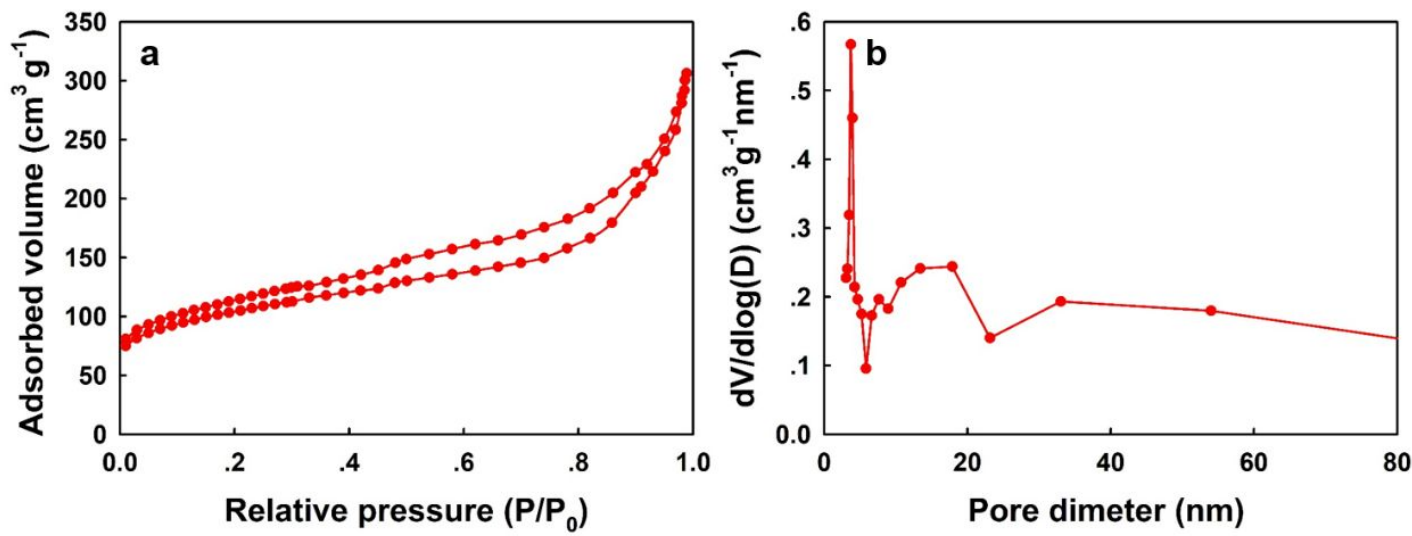

Figure S11. $\mathrm{N}_{2}$ sorption isothermals (a) and pore size distribution (b) of rGO@mCMnO-800 

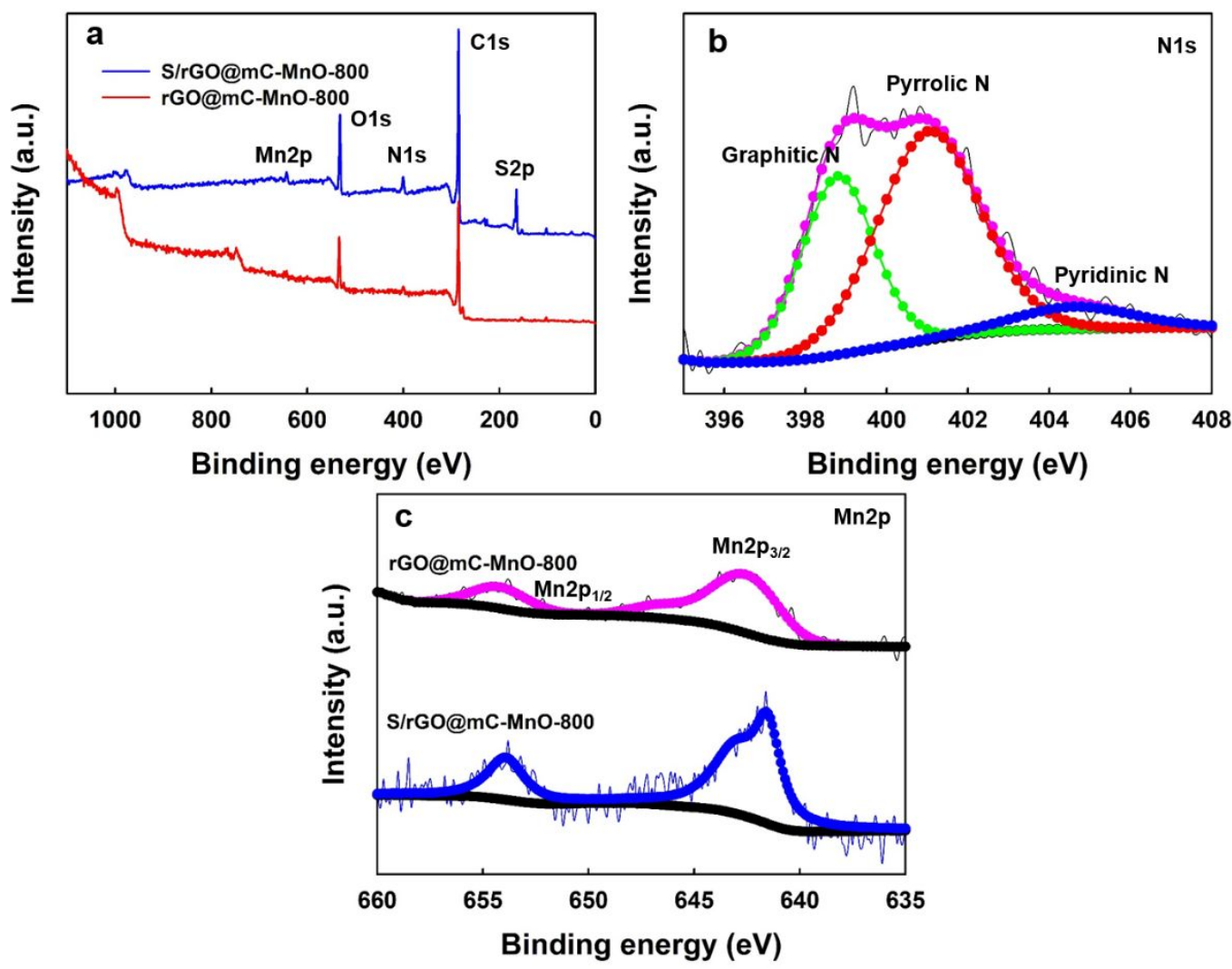

Figure S12. Total XPS survey spectra of rGO@mC-MnO-800 and S/rGO@mC-MnO800 (a); The N 1s XPS spectrum of the rGO@mC-MnO-800; The Mn2p XPS spectrum of rGO@mC-MnO-800 and S/rGO@mC-MnO-800. 


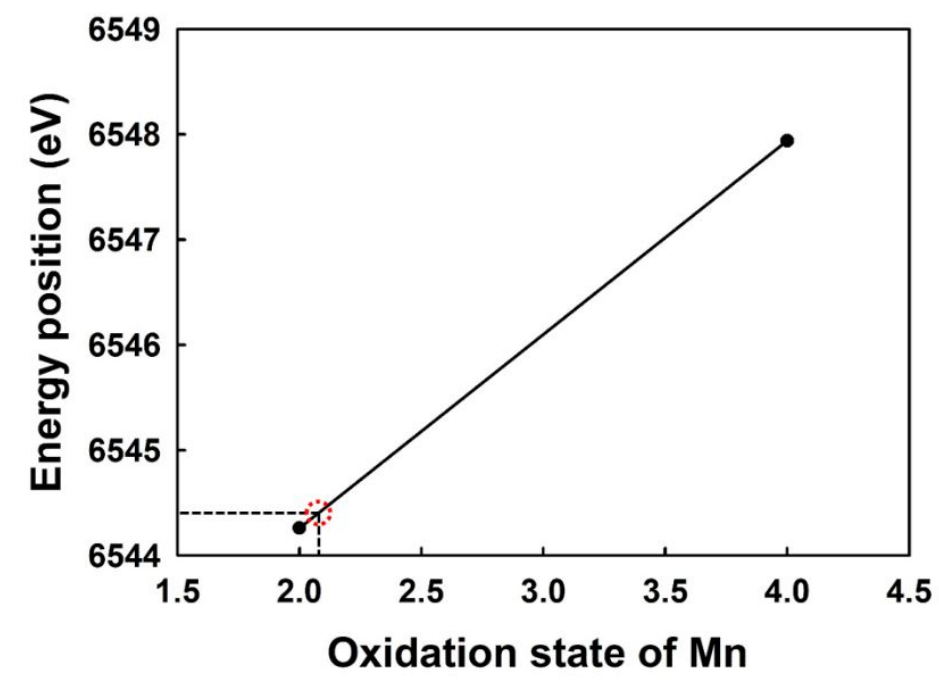

Figure S13. The oxidation state fitting of Mn element in the rGO@mC-MnO-800 sample by using the edge energy of standard samples. 
a

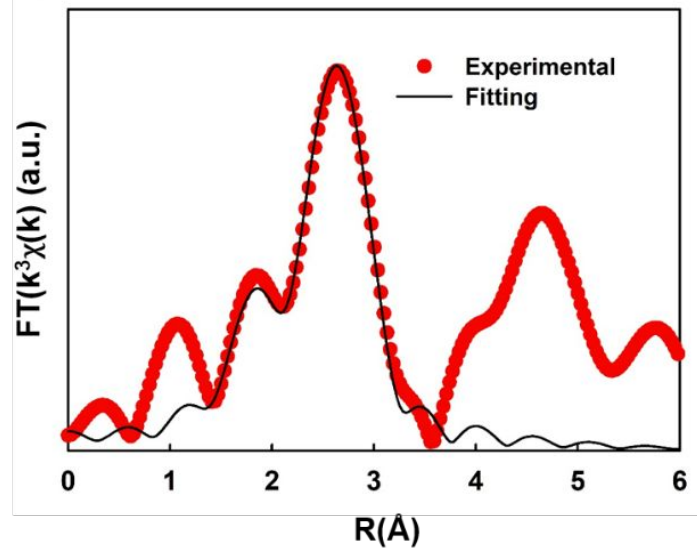

b

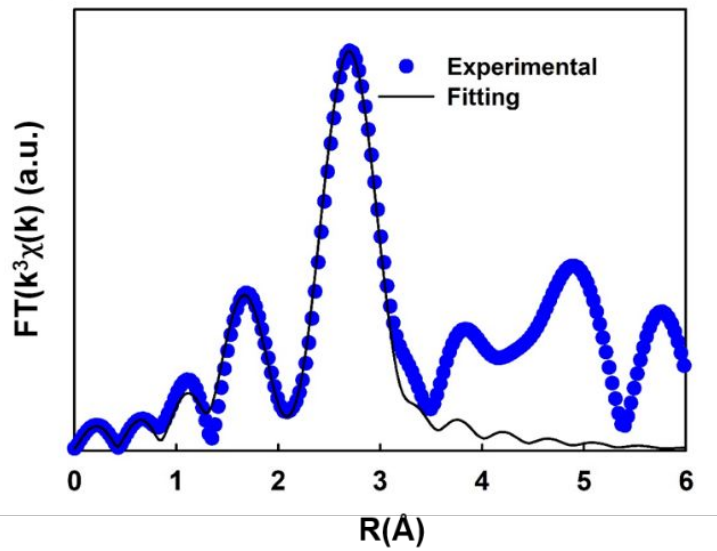

Figure S14. Quantitative EXAFS curves fitting at R-space for the rGO@mC-MnO-800

(a) and rGO@mC-MnO-900 (b). 

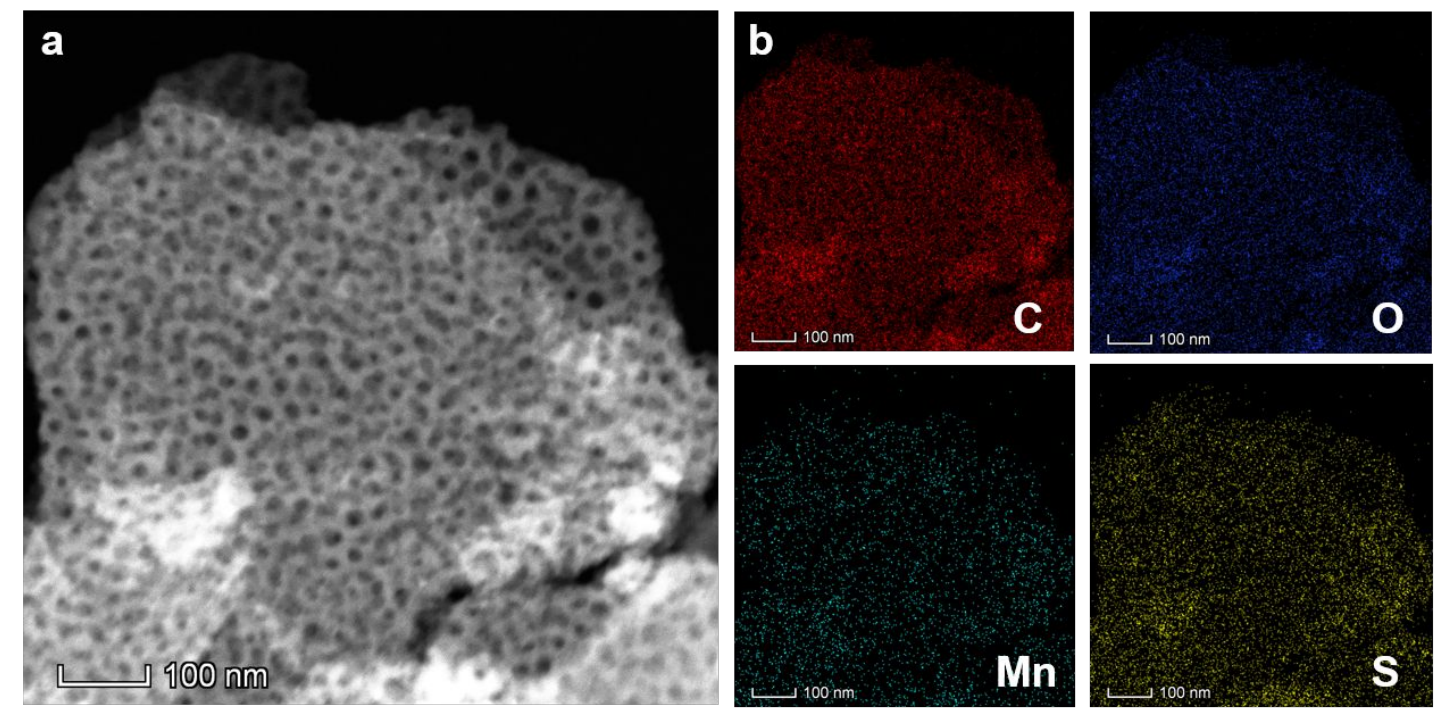

Figure S15. STEM image of S/rGO@mC-MnO-800 (a) and the corresponding elemental maps (b) of carbon, oxygen, manganese, and sulfur. 

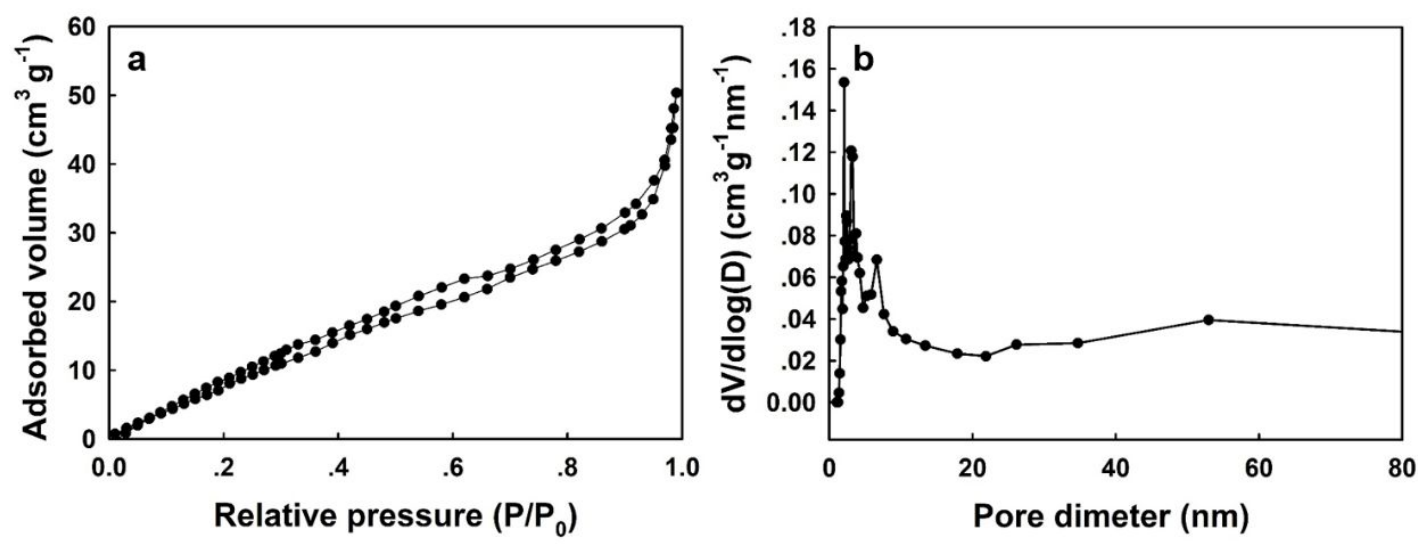

Figure S16. $\mathrm{N}_{2}$ sorption isothermals (a) and pore size distribution (b) of S/rGO@mC-

MnO-800. 


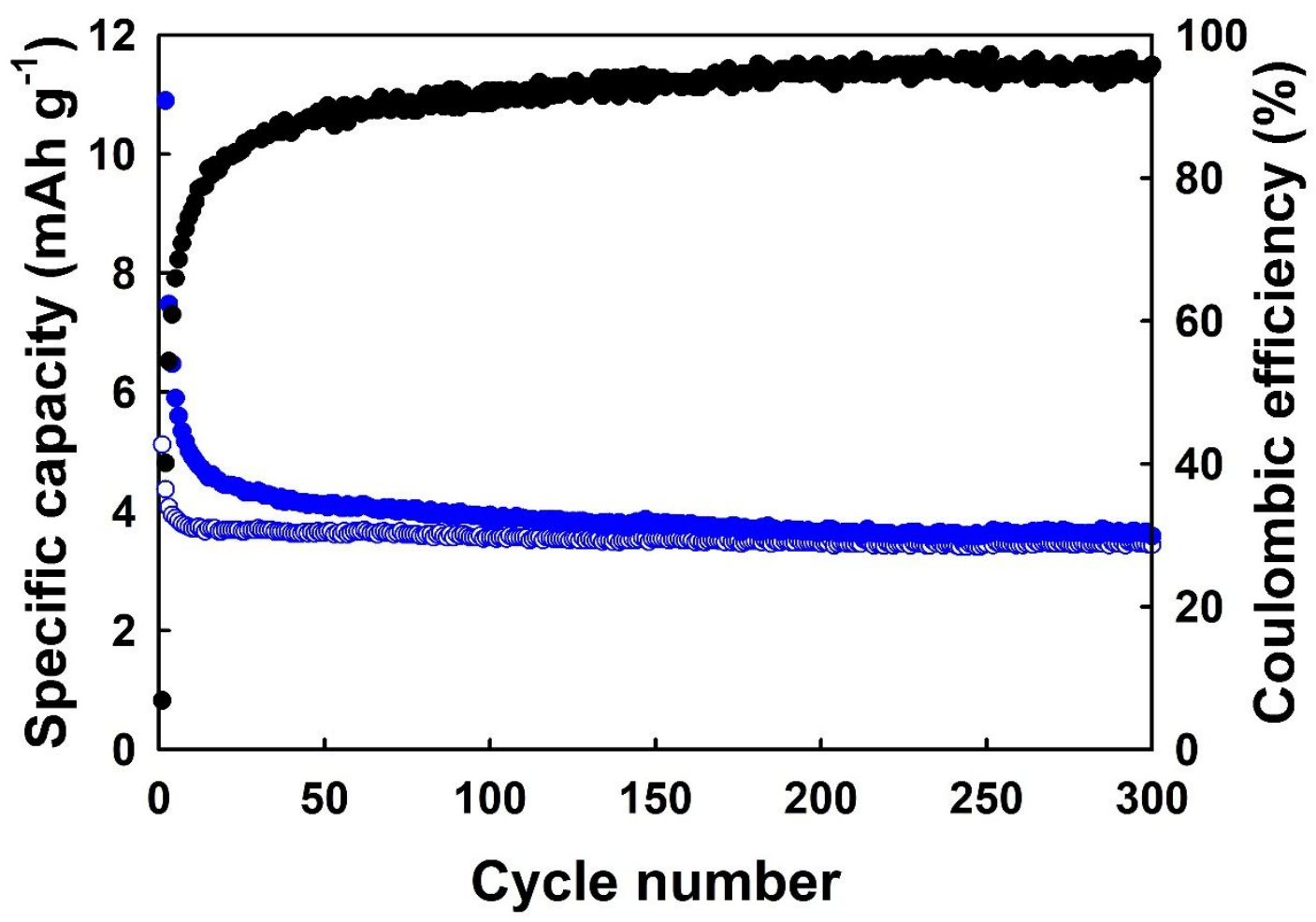

Figure S17. The electrochemical performance of rGO@mC-MnO-800 cathode at 0.2 $\mathrm{A} \mathrm{g}^{-1}$. 

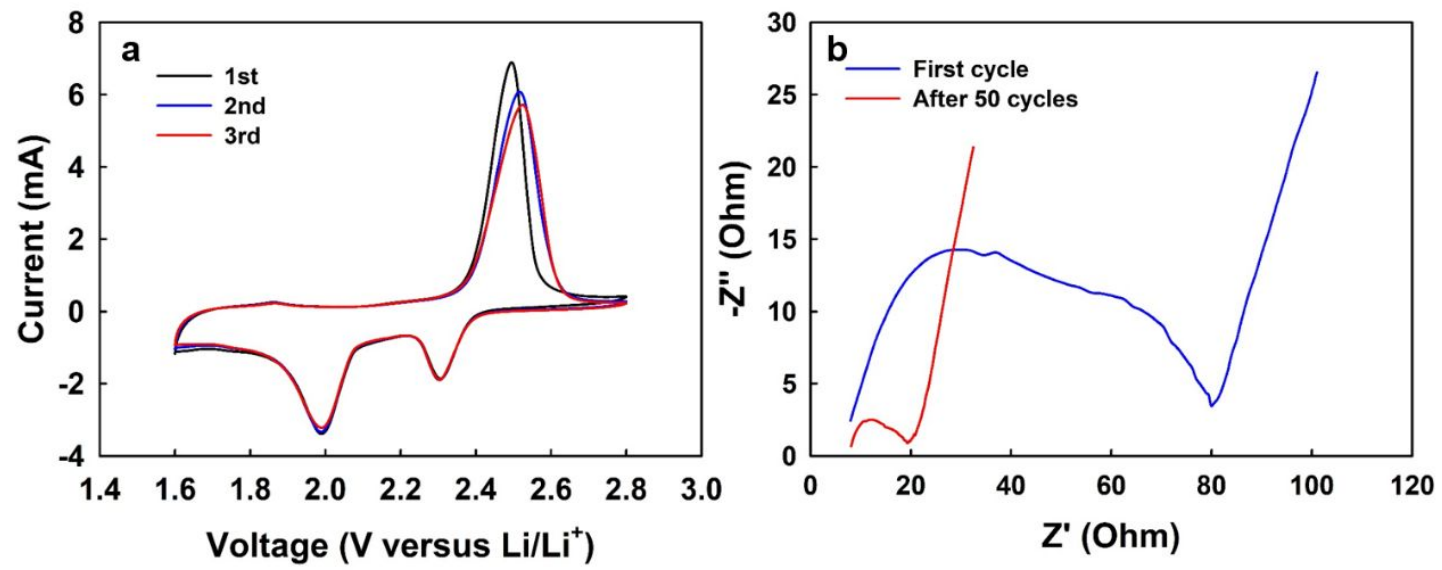

Figure S18. The cyclic voltammogram curves of the S/rGO@mC-MnO-800 with a scan rate of $0.2 \mathrm{mV} \mathrm{s}^{-1}$ (a) and electrochemical impedance spectroscopy (EIS) analysis of the S/rGO@mC-MnO-800 at the first and 50 cycles (b). 


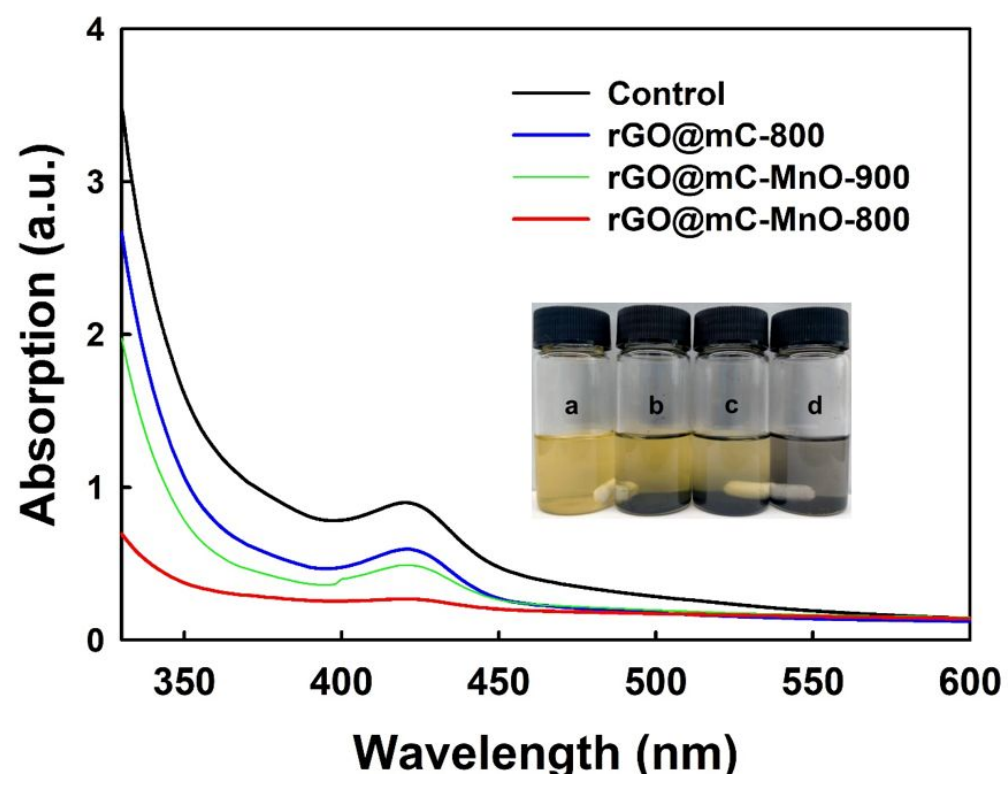

Figure S19. The UV-vis spectra of different samples (a) control, (b) rGO@mC-800, (c) rGO@mC-MnO-900 and (d) rGO@mC-MnO-800 after soaking in the $\mathrm{Li}_{2} \mathrm{~S}_{6}$ solution for $24 \mathrm{~h}$. The insert shows their corresponding digital photos. 
a

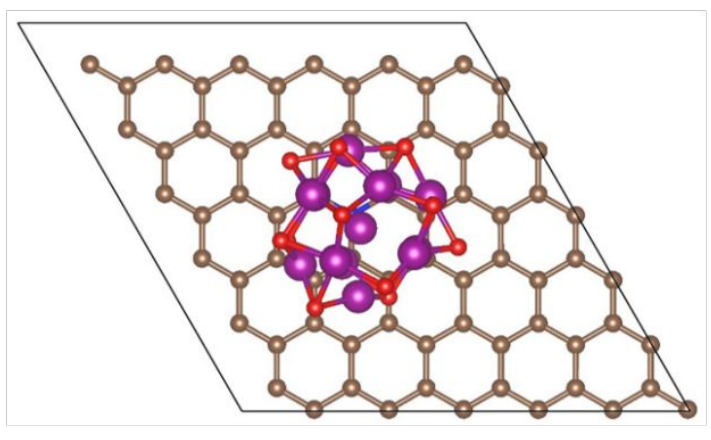

b

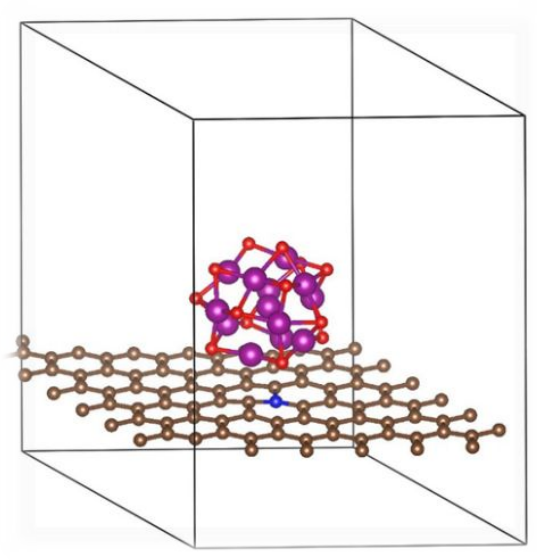

Figure S20. Schematic illustration of a $\mathrm{Mn}_{13} \mathrm{O}_{13}$ cluster adsorbed on N-doped graphene substrate. Top view (a) and side view (b). 
Table S1. The XANES curve-fitting results of rGO@mC-MnO-800 and rGO@mC$\mathrm{MnO}-900$.

\begin{tabular}{|c|c|c|c|c|c|c|}
\hline \multirow{2}{*}{$\begin{array}{l}\text { Sample } \\
\text { name }\end{array}$} & \multirow[b]{2}{*}{ Coordination } & \multirow[b]{2}{*}{$\mathrm{N}$} & \multicolumn{3}{|c|}{$\sigma^{2} \times 10^{-3}$} & \multirow{2}{*}{$\begin{array}{c}\text { R- } \\
\text { factor }\end{array}$} \\
\hline & & & $\mathrm{R}(\AA)$ & $\left(\AA^{2}\right)$ & $\Delta \mathrm{E}(\mathrm{eV})$ & \\
\hline \multirow{3}{*}{$\mathrm{MnO}$} & $\mathrm{Mn}-\mathrm{O}$ & 6 & 2.22 & - & - & \multirow{3}{*}{-} \\
\hline & & & & & & \\
\hline & $\mathrm{Mn}-\mathrm{Mn}$ & 12 & 3.14 & - & - & \\
\hline \multirow{2}{*}{$\mathrm{MnO}_{2}$} & $\mathrm{Mn}-\mathrm{O}$ & 6 & 1.99 & & & \\
\hline & $\mathrm{Mn}-\mathrm{Mn}$ & 6 & 3.10 & & & \\
\hline rGO@mC- & $\mathrm{Mn}-\mathrm{O}$ & $1.3 \pm 0.4$ & $2.26 \pm 0.02$ & 7.0 & 3.0 & \multirow[b]{2}{*}{$1.85 \%$} \\
\hline $\mathrm{MnO}-800$ & $\mathrm{Mn}-\mathrm{Mn}$ & $2.9 \pm 1.2$ & $3.11 \pm 0.03$ & $7.8 \pm 3.9$ & $-7.1 \pm 3.4$ & \\
\hline rGO@mC- & $\mathrm{Mn}-\mathrm{O}$ & $3.0 \pm 1.4$ & $2.22 \pm 0.05$ & $6.1 \pm 3.0$ & $-1.7 \pm 1.1$ & \multirow[b]{2}{*}{$1.24 \%$} \\
\hline MnO-900 & $\mathrm{Mn}-\mathrm{Mn}$ & $6.7 \pm 0.8$ & $3.13 \pm 0.02$ & $7.0 \pm 2.6$ & $-5.3 \pm 2.4$ & \\
\hline
\end{tabular}

$\mathrm{N}$, coordination number; $\mathrm{R}$, distance between $\mathrm{Mn}$ and $\mathrm{O} / \mathrm{Mn}$ atoms; $\sigma^{2}$, change in the Debye-Waller factor value relative to the Debye-Waller factor of the reference compound; $\Delta \mathrm{E}$, inner potential correction to account for the difference in the inner potential between the sample and the reference compound; R, the correction coefficient.

\section{Reference}

[1] Yu, H. S.; Wei, X. J.; Li, J.; Gu, S. Q.; Zhang, S.; Wang, L. H.; Ma, J. Y.; Li, L. N.; Gao, Q.; Si, R.; Sun, F. F.; Wang, Y.; Song, F.; Xu, H. J.; Yu, X. H.; Zou, Y.; Wang, J. Q.; Jiang, Z. H.; Huang, Y. Y. The XAFS beamline of SSRF, Nucl. Sci. Tech. 2015, 26 (5), 410. 
[2] Newville, M. IFEFFIT: interactive XAFS analysis and FEFF fitting. J. Synchrotron Rad. 2001, 8, 322-324.

[3] Kresse, G.; Furthmuller, J. Efficient iterative schemes for ab initio total-energy calculations using a plane-wave basis set. Phys. Rev. B 1996, 54 (16), 11169.

[4] Kresse, G.; Furthmuller, J. Efficiency of ab-initio total energy calculations for metals and semiconductors using a plane-wave basis set. Comput. Mater. Sci. 1996, 6 (1), 15-50.

[5] Blöchl, P. E. Projector augmented-wave method. Phys. Rev. B 1994, 50 (24), 17953. [6] Perdew, J. P.; Burke, K.; Ernzerhof, M. Generalized gradient approximation made simple. Phys. Rev. Lett. 1996, 77 (18), 3865.

[7] Booth, C. H.; Durakiewicz, T.; Capan, C.; Hurt, D.; Bianchi, A. D.; Joyce, J. J.; Fisk, Z. Electronic structure and f-orbital occupancy in Yb-substituted CeCoIn 5 . Phys. Rev. B 2011, 83 (23), 235117.

[8] Dion, M.; Rydberg, H.; Schröder, E.; Langreth, D. C.; Lundqvist, B. I. Erratum: Van der Waals Density Functional for General Geometries. Phys. Rev. Lett. 2004, 92 (10), 246401.

[9] Hamada, I. Van der Waals density functional made accurate. Phys. Rev. B 2014, 89 (12), 121103. 